\title{
Multidrug-Resistant and Carbapenemase-Producing Enterobacteriaceae in Addis Ababa, Ethiopia
}

\author{
Saba Gebremichael Tekele $\mathbb{D}^{1},{ }^{1}$ Dejenie Shiferaw Teklu $\mathbb{D}^{2}{ }^{2}$ Melese Hailu Legese, ${ }^{3}$ \\ Daniel Gebretsadik Weldehana $\mathbb{D}^{1},{ }^{1}$ Melaku Ashagrie Belete $\mathbb{\infty},{ }^{1}$ Kassu Desta Tullu, ${ }^{3}$ \\ and Samuel Kinde Birru ${ }^{3}$ \\ ${ }^{1}$ Department of Medical Laboratory Sciences, College of Medicine and Health Sciences, Wollo University, Dessie, Ethiopia \\ ${ }^{2}$ Department of Bacteriology and Mycology, Ethiopian Public Health Institute, Addis Ababa, Ethiopia \\ ${ }^{3}$ Department of Medical Laboratory Sciences, College of Health Sciences, Addis Ababa University, Addis Ababa, Ethiopia \\ Correspondence should be addressed to Saba Gebremichael Tekele; sabagmichael2@gmail.com
}

Received 11 March 2021; Revised 31 May 2021; Accepted 1 June 2021; Published 12 June 2021

Academic Editor: Stefania Cantore

Copyright @ 2021 Saba Gebremichael Tekele et al. This is an open access article distributed under the Creative Commons Attribution License, which permits unrestricted use, distribution, and reproduction in any medium, provided the original work is properly cited.

\begin{abstract}
Background. The emergence and spread of multi-drug resistant (MDR) bacteria have become a public health problem in recent years. For the last many years, carbapenem antibiotics have been used successfully to treat infections caused by MDR Enterobacteriaceae. However, recently, Enterobacteriaceae producing carbapenemases have emerged, which confer broad resistance to most $\beta$-lactam antibiotics including carbapenems. Therefore, this study is aimed at determining the magnitude of MDR and carbapenemase-producing Enterobacteriaceae (CPE) isolated from various clinical specimens in Addis Ababa, Ethiopia. Methods. A cross-sectional study was conducted from January to April 2018. A total of 312 Enterobacteriaceae isolates were identified from various clinical specimens. The Phoenix automated system (BD Phoenix100) was used for bacterial identification and antimicrobial susceptibility testing. Potential carbapenemase producers were confirmed by the modified carbapenem inactivation test, and KPC, MBL, and OXA-48 were phenotypically characterized by the disk diffusion method. The data obtained were entered and analyzed using SPSS version 20 software. Descriptive statistics, chi square, bivariate and multivariable logistic regression analyses were performed. $P$ value $\leq 0.05$ with corresponding $95 \%$ confidence interval was considered for statistical significance. Results. A total of 312 Enterobacteriaceae were recovered. Of these isolates, $68.6 \%$ were MDR and 2.6\% were CPE with different classes including OXA-48 1.6\% (5/312), MBL 0.6\% (2/312), and KPC and OXA-48 $0.3 \%$ (1/312). The predominant bacterial isolates were E. coli $72.4 \%$ (226/312) followed by K. pneumoniae 13.8\% (43/312). The antibiotic resistance rates of CPE isolates were significantly higher than other MDRE including ampicillin (100\% versus $77.6 \%)$, cefoxitin (75\% versus $20.6 \%$ ), and piperacillin/tazobactam (50\% versus $13.1 \%)$. Conclusion. In this study, a relatively higher prevalence of MDR was observed, and the highest resistance was recorded against ampicillin, amoxicillin with clavulanic acid, and sulfamethoxazole-trimethoprim. Detection of CPE is important for implementing appropriate antimicrobial therapy and in controlling the spread of the infection. Furthermore, continuous screening and investigations, including genotypic characterization of $\mathrm{CPE}$, are required for the prevention and control of the spread of antimicrobial-resistant pathogens.
\end{abstract}

\section{Background}

The emergence and spread of multi-drug resistant (MDR) bacteria have become a public health problem in recent years [1]. Of particular concern are infections caused by resistant Enterobacteriaceae, which are common pathogens causing different types of community- and hospital-acquired infec- tions, and antimicrobial resistance in these bacteria has significant impacts on patient outcomes [2].

For the last many years, carbapenem antibiotics have been used successfully to treat infections caused by multidrug resistant Enterobacteriaceae, including those producing extended spectrum $\beta$-lactamases (ESBL). However, recently, Enterobacteriaceae producing carbapenemases have 
emerged, which confer broad resistance to most $\beta$-lactam antibiotics including "last-line" carbapenems [1].

Even though there are several mechanisms for the resistance of carbapenem such as a decrease in permeability of bacterial outer membrane with overexpression of Amp$\mathrm{C} / \mathrm{ESBL}$ or efflux pump, the main mechanism of carbapenem resistance is the production of carbapenemase [3]. The most common carbapenemases include veronica integron metallo$\beta$-lactamase types (VIM), imipenemase (IMP), K. pneumoniae carbapenemase (KPC), oxacillinase-48 (OXA-48), and New Delhi metallo- $\beta$-lactamase-1 (NDM-1) [4]. These carbapenemase genotypes differed geographically. For instance, OXA-48 enzymes particularly recovered within the Mediterranean area, including Northern Africa and Middle East [5].

According to the World Health Organization (WHO) recent report, CPE has listed among the antibiotic-resistant bacteria with a level one (critical) priority for research and development of new antibiotics [6].

The prevalence of carbapenem-resistant Enterobacteriaceae (CRE) infections has increased over the last decade, especially in healthcare settings, and the Centers for Disease Control and Prevention (CDC) estimates that more than 9000 healthcare-associated infections are caused by CRE each year in the United States [7, 8]. Moreover, CRE can cause a number of serious infections such as intra-abdominal infections, pneumonia, urinary tract infections, and deviceassociated infections [9-11]. The mortality rates are also high and range from $18 \%$ to $48 \%$ depending on therapy [12].

Currently in Ethiopia, different studies showed that there is increasing use of carbapenem in health facilities or physicians use carbapenem for empirical treatment. Due to this, treatment options for serious CRE infections remain limited. Optimization of dosing of currently available agents and combination therapy may be the most appropriate treatment strategies at this time. However, continued research is desperately needed, in particular randomized controlled trials, to determine the most appropriate treatment for serious CRE infections. Therefore, this study is aimed at determining the magnitude of MDR and carbapenemase-producing Enterobacteriaceae isolated from various clinical specimens in Addis Ababa, Ethiopia.

\section{Methods}

A cross-sectional study was conducted at International Clinical Laboratories (ICL) in Addis Ababa, Ethiopia, from January 1 to April 30, 2018. A total of 312 Enterobacteriaceae isolates were recovered from different clinical specimens and included using a convenient sampling technique for carbapenemase characterization and antimicrobial susceptibility testing. A predeveloped worksheet was used to collect information related to sociodemographic characteristics of the patients. The identified Enterobacteriaceae isolates, the antibiotic susceptibility pattern of the isolate, and classes of carbapenemases were also recorded using a separate data collection sheet.

2.1. Cultivation and Identification of Isolates. Different clinical specimens were inoculated onto appropriate culture media (sheep blood agar, XLD agar, and MacConkey agar plates (Oxoid Ltd, UK)) and incubated overnight under the aerobic condition at $37^{\circ} \mathrm{C}$ for $18-24$ hours. Identification of Enterobacteriaceae was done using colony characteristics, Gram staining reaction, and ability to ferment lactose. In addition, the Phoenix system (BD Diagnostic Systems, Oxford, UK) was used for the identification of the bacteria to species level. The combination panel includes identification (ID) side with dried substrates for bacterial identification, and the instrument tests panels every 20 minutes. After three hours, the identified bacteria were displayed on the screen of the Phoenix system [13].

2.2. Antimicrobial Susceptibility Testing. Antimicrobial susceptibility testing was performed using the Phoenix AST panel (AST-N94). The following antimicrobials were included: ceftazidime, cefuroxime, ciprofloxacin, ceftriaxone, cefepime, amoxicillin with clavulanic acid, amikacin, aztreonam, ertapenem, cefoxitin, gentamicin, imipenem, meropenem, ampicillin, sulfamethoxazole-trimethoprim, and piperacillin/tazobactam [13]. Results were interpreted according to CLSI recommendations [14]. Multi-drug resistant isolates were determined using the definition of Magiorakos et al. [15].

2.3. Carbapenemase Detection. Potential carbapenemaseproducing isolates, which showed resistance to at least one of the tested carbapenems (ertapenem, imipenem, and meropenem) were selected when MIC $\geq 2 \mu \mathrm{g} / \mathrm{ml}$ for imipenem, $\geq 1$ $\mu \mathrm{g} / \mathrm{ml}$ for ertapenem, and/or $\geq 2 \mu \mathrm{g} / \mathrm{ml}$ for meropenem [13].

Suspected carbapenemase-producing isolates were confirmed by the modified carbapenem inactivation methods (mCIM) which are recommended by CLSI [14]. After preparation of bacterial suspension of tested isolates in $2 \mathrm{ml}$ Trypticase soya broth, meropenem disk $(10 \mu \mathrm{g})$ was added and incubated at $35^{\circ} \mathrm{C} \pm 2^{\circ} \mathrm{C}$ in ambient air for 4 hours \pm 15 minutes. When the time was completed, meropenem disk $(10 \mu \mathrm{g})$ was placed on a Muller Hinton agar plate inoculated with E. coli ATCC 25922 suspension of $0.5-\mathrm{McF}$ arland turbidity standards and incubated overnight (18-24hrs.) at $37^{\circ} \mathrm{C}$. Bacterial isolates having a zone of inhibition 6-15 $\mathrm{mm}$ or presence of pinpoint colonies within a $16-18 \mathrm{~mm}$ zone and no inhibition of the meropenem-susceptible E. coli ATCC 25922 were confirmed as carbapenemase-producing isolates.

2.4. Phenotypic Characterization of Carbapenemases. Carbapenemases were characterized phenotypically by a disk diffusion method using Neo-Sensitabs disks. The organism to be tested was spread onto a Mueller Hinton agar plate using similar procedures as for drug susceptibility testing. A meropenem $(10 \mu \mathrm{g})$ disk alone and in combination with inhibitors of different beta-lactamases such as phenylboronic acid (KPC and AmpC inhibitor), dipicolinic acid (MBL inhibitor), cloxacillin (AmpC inhibitor), and temocillin disks $(30 \mu \mathrm{g})$ (Rosco, Taastrupgaardsvej, Denmark) was used; then, the organism was incubated at $37^{\circ} \mathrm{C}$ for 24 hours. An increase in the inhibition zone diameter for a combination disk versus meropenem disk alone was interpreted as a carbapenemase producer [16]. 
TABLE 1: Distribution of Enterobacteriaceae isolate among various specimen types at ICL, Addis Ababa, Ethiopia.

\begin{tabular}{|c|c|c|c|c|c|c|c|}
\hline & Urine & Pus & Body fluid & Ear discharge & Eye discharge & Stool & Total \\
\hline E. coli & $192(84.9)$ & $25(11.1)$ & $7(3.1)$ & $1(0.4)$ & $2(0.9)$ & $0(0.0)$ & 226 \\
\hline K. pneumoniae & $26(60.5)$ & $12(27.9)$ & $2(4.7)$ & $1(2.3)$ & $1(2.3)$ & $0(0.0)$ & 43 \\
\hline Enterobacter spp. & $5(45.5)$ & $6(54.5)$ & $0(0.0)$ & $0(0.0)$ & $0(0.0)$ & $0(0.0)$ & 11 \\
\hline Citrobacter spp. & $9(81.8)$ & $2(18.2)$ & $0(0.0)$ & $0(0.0)$ & $0(0.0)$ & $0(0.0)$ & 11 \\
\hline P. mirabilis & $2(33.3)$ & $2(33.3)$ & $0(0.0)$ & $2(33.3)$ & $0(0.0)$ & $0(0.0)$ & 6 \\
\hline Shigella spp. & $0(0.0)$ & $0(0.0)$ & $0(0.0)$ & $0(0.0)$ & $0(0.0)$ & $2(100)$ & 2 \\
\hline Other isolates & $7(53.8)$ & $5(38.5)$ & $1(7.7)$ & $0(0.0)$ & $0(0.0)$ & $0(0.0)$ & 13 \\
\hline Total & 241 & 52 & 10 & 4 & 3 & 2 & 312 \\
\hline
\end{tabular}

Note: *other isolates are Salmonella spp, Providencia spps, M. morganii, and Serratia spp.

2.5. Quality Assurance. To maintain the quality of the work from isolate collection up to final bacterium identification and data management, the standard operating procedure of isolate collection and laboratory analysis was strictly followed. The expiry date of the media, reagents, and antibiotic disks was checked before use. The prepared culture media was checked for sterility. Abilities of the prepared media supporting the growth of organisms were checked by inoculating ATCC control strain including S. aureus (ATCC 25923), E. coli (ATCC 25922), E. faecalis (ATCC 29212), and P. aeruginosa (ATCC 27853) [14]. Quality control testing for the Phoenix machine was done for each lot of panels [13]. For carbapenemase detection, BAA1705 control strains as positive control and BAA1706 as negative control were used [16].

2.6. Data Analysis and Interpretation. Data was collected using a worksheet and analysed using SPSS version 20 software. Descriptive statistics, chi square, and bivariate and multivariable logistic regression analyses were performed. $P$ value $\leq 0.05$ with corresponding $95 \%$ confidence interval was considered for statistical significance.

Frequency as well as percentages of MDR and carbapenemase-producing Enterobacteriaceae was calculated. Moreover, data was presented using tables and graphs.

\section{Results}

3.1. Magnitude of Enterobacteriaceae. A total of 312 Enterobacteriaceae isolates were isolated from various clinical specimens sent to the microbiology laboratory. Of these isolates, $58.0 \%(n=181 / 312)$ were from females, while $42.0 \%$ $(n=131 / 312)$ were from males with a mean (standard deviation) age of $44.2(21.8)$ years, and $67.3 \%(n=210 / 312)$ of them were inpatients. About 77.2\% (241/312) of Enterobacteriaceae were isolated from urine, and 16.7\% (52/312) were from pus. Among Enterobacteriaceae isolates, E. coli was the dominant isolate accounting for $72.4 \%$ (226/312), and $K$. pneumoniae was the second predominant species representing $13.8 \%(43 / 312)$ of the total isolates. About $84.9 \%$ $(192 / 226)$ of $E$. coli was isolated from urine, and $11.1 \%$ $(25 / 226)$ were from pus specimens (Table 1$)$.

3.2. Multi-drug Resistance Patterns of Enterobacteriaceae. Out of the total 312 Enterobacteriaceae isolate enrolled in this study, 68.6\% (214/312) were MDR. The principal MDR isolates were Enterobacter spp. (90.9), Citrobacter spp. (81.8), K. pneumoniae (79.1), and E. coli (68.8) (Table 2).

3.3. Carbapenemase-Producing Enterobacteriaceae. From the total isolates, 17 isolates were potential carbapenemase producers. Of these isolates, $23.5 \%(8 / 17)$ was confirmed as a carbapenemase producer by mCIM. The overall magnitude of carbapenemase-producing Enterobacteriaceae was $2.6 \%$ (8/312) which includes K. pneumoniae 1.3\% (4/312), E. coli 0.9\% (3/312), and Enterobacter spp. 0.3\% (1/312).

Similarly, those confirmed carbapenemase-producing Enterobacteriaceae were phenotypically characterized by a combination disk test using Neo-Sensitabs ${ }^{\mathrm{TM}}$ (Rosco, Denmark), and OXA-48 1.6\% (5/312), MBL 0.6\% (2/312), and KPC and OXA-48 0.3\% (1/312) were identified classes of carbapenemases (Table 3 ).

3.4. Antibiotic Resistance Pattern of MDRE and CPE. The majority of isolated MDR Enterobacteriaceae showed a resistance level of $92.5 \%$ for amoxicillin with clavulanic acid followed by $77.6 \%$ for ampicillin and $75.7 \%$ for sulfamethoxazole-trimethoprim. Among the isolates, E. coli showed the highest resistance to ampicillin (98.0\%) followed by amoxicillin with clavulanic acid (93.4\%). The second most common isolate K. pneumoniae showed $82.4 \%$ to amoxicillin with clavulanic acid, $85.3 \%$ to sulfamethoxazole-trimethoprim, and $76.5 \%$ to ceftriaxone. However, all isolates showed a relatively low level of resistance against amikacin (1.4\%), meropenem (3.3\%), and imipenem (4.7\%) (Table 4).

The antibiotic resistance rates of CPE isolates were significantly higher than other MDRE including ampicillin (100\% versus $77.6 \%$ ), cefoxitin (75\% versus $20.6 \%$ ), and piperacillin/tazobactam (50\% versus $13.1 \%)$. As shown in Figure 1, $100 \%$ resistance was observed to ampicillin and amoxicillin-clavulanic acid. Moreover, only 25\% of strains were resistant to ciprofloxacin, and no resistance was observed to amikacin.

3.5. Carbapenemase-Producing Enterobacteriaceae and MDR Level among Different Specimens. Among specimens investigated, MDR-producing Enterobacteriaceae were found predominantly in body fluid 100\% (10/10) whereas majority of carbapenemase-producing Enterobacteriaceae were isolated 


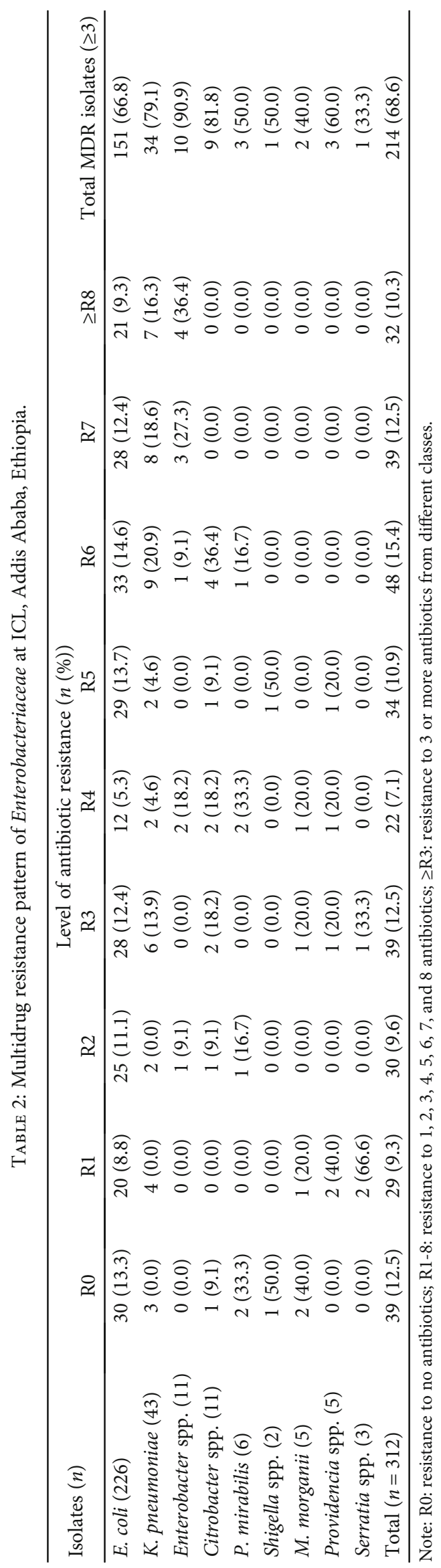


TABLE 3: Distribution of carbapenemase-producing Enterobacteriaceae at ICL, Addis Ababa, Ethiopia.

\begin{tabular}{lcccc}
\hline Isolates (number) & mCIM & OXA-48 & MBL & KPC+OXA-48 \\
\hline E. coli (226) & $3(1.3)$ & $3(1.3)$ & $0(0.0)$ & $0(0.0)$ \\
K. pneumoniae (43) & $4(9.8)$ & $2(4.9)$ & $1(2.4)$ & $1(2.4)$ \\
Enterobacter Spp. (11) & $1(9.1)$ & $0(0.0)$ & $1(9.1)$ & $0(0.0)$ \\
Citrobacter Spp. $(11)$ & $0(0.0)$ & $0(0.0)$ & $0(0.0)$ & $0(0.0)$ \\
P. mirabilis (6) & $0(0.0)$ & $0(0.0)$ & $0(0.0)$ & $0(0.0)$ \\
Shigella Spp. (2) & $0(0.0)$ & $0(0.0)$ & $0(0.0)$ & $0(0.0)$ \\
Other Spp. $(13)$ & $0(0.0)$ & $0(0.0)$ & $0(0.0)$ & $0(0.0)$ \\
Total $(n=312)$ & $8(2.4)$ & $5(1.5)$ & $2(0.6)$ & $1(0.3)$ \\
\hline
\end{tabular}

from urine 2.1\% (5/241) (Figure 2). All carbapenemase producing Enterobacteriaceae were MDR.

3.6. Association of Independent Variables with MDRE. In multivariable analysis, the odds of having MDRE were 8.82 times $(\mathrm{AOR}=8.824,95 \% \mathrm{CI}:(3.769,20.654), P<0.001)$ more likely among inpatients than outpatients. Moreover, the remaining variables such as age, sex, and specimen types do not have statistically significant association with MDRE.

\section{Discussion}

Beta-lactamase-producing Enterobacteriaceae have become a global threat. Production of carbapenemase with the emergence of antibacterial resistance is the most important cause of empirical treatment failures. Moreover, current knowledge of the prevalence of MDR Gram-negative bacteria is important to understand their epidemiology and the disease burden and also to strengthen hospital infection control strategy [3].

In this study, MDR (nonsusceptible to $\geq 1$ agent in $\geq 3$ antimicrobial categories) was observed in $68.6 \%$. This finding was in line with studies conducted in Addis Ababa (68.3\%) [17], Gondar (68.0\%) [18], Debre Markos (72.2\%) [19], and Nepal (64.0\%) [20] while it was lower than studies done in Ethiopia such as Addis Ababa (94.5\%) [21], Gondar (87.4\%) [22], and Bahir Dar (93.1\%) [23] and studies done in Mozambique (88.2\%) [24], Sierra Leone (85.7\%) [25], Iran (91.5\%) [26], and Nepal (96.8\%) [27]. The reason for the variation might be due to differences in the AST method and the presence of beta-lactamase-producing organisms which are resistant to multiple classes of antibiotics. Compared with the present study, MDR isolates were lower in Jimma (59.3\%) [28], Italy (62.0\%) [29], Nepal (54.2\%) [20], and USA (19.1\%) [30]. The difference in the magnitude of MDR might be due to the definition used to classify isolates into MDR, patient condition, and presence of carbapenemase-producing isolates in these studies. Repeated, inappropriate, and incorrect use of antimicrobial agents in empirical treatment and poor infection control strategies, in turn, raise the prevalence of resistant bacteria in the community.

The highest MDR strains were detected from Enterobacter spp. (90.9\%) and Citrobacter spp (81.8\%) which is comparable to other studies done in Jimma [28] and Nepal [31].
Different studies showed different pathogens as predominant MDR isolates, K. pneumoniae and E. coli in Gondar [22], Sierra Leone [24], Iran [32], and Nepal [20]. The difference might be due to these bacteria being found in both hospitaland community-acquired infections. In addition, these bacteria are resistant to multiple groups of antimicrobial agents which makes treatment difficult [20].

Although no nationwide study has been conducted so far for the detection of carbapenemase-producing Enterobacteriaceae in Ethiopia, some studies have been done in some parts of the country $[19,21,22]$.

The present study showed that out of seventeen carbapenemase-suspected Enterobacteriaceae, 8 (2.6\%) were carbapenemase producers which was in line with a study done in Addis Ababa (2\%) [21], Gondar (2.73\%) [22], Morocco (2.8\%) [33], Taiwan (2.5\%) [34], and Jordan (2.8\%) [35]. The magnitude of carbapenemase-producing Enterobacteriaceae in the current study was lower when compared with the finding in Addis Ababa 12.12\% [36], Uganda $22.4 \%$ [37], India 23\% [38], and Sudan 56\% [39]. The difference in these findings might be due to method difference and the patient condition (in which others only include inpatient and isolates resistant to at least two 3GC (third-generation cephalosporin)). Furthermore, the variation might be due to the difference in local antibiotic prescribing habits and infection control program in different health facilities [39].

In this study, OXA-48 enzyme was the most prevalent carbapenemase in Addis Ababa which was supported by a study done by Manenzhe et al. reporting that oxacillinases especially OXA-48 were the most predominant type of carbapenemase in Africa [40]. Studies in Egypt [41] and Spain [42] also showed similar findings. Prevalence and types of carbapenemases can be affected by the difference in phenotypic methods, difference in the study area, and prevalence of carbapenemase genes in different countries [39]. However, surveillance, hand hygiene, and appropriate antibiotic usage are part of an effective approach in reducing the dissemination of these pathogenic organisms [43].

Co-production of KPC and OXA-48 enzymes in the current study was found in one carbapenem-resistant isolate. A comparable result was reported in Uganda [37], but this finding was not in agreement with the finding in Nigeria [44] and Thailand [45] which showed coproduction of NDM and KPC enzyme in one isolate, this is because NDM was the predominant enzyme in these countries. Co-production of two carbapenemase enzymes by one bacterium results in the inactivation of beta-lactamase inhibitors and high-level resistance to the carbapenems as well [42].

The present study showed that the most common carbapenemase-producing Enterobacteriaceae were K. pneumonia which was agreed with a study done in Addis Ababa [36], Thailand [45], and Jordan [35]. However, it was inconsistent compared to the study done in Gondar [22], Sudan [39], and Pakistan [46] indicating that the principal carbapenemaseproducing pathogen was E. coli than K. pneumonia.

Majority of carbapenemase-producing Enterobacteriaceae in the present study was isolated from urine specimens. This agrees with the previous studies conducted in Ethiopia, Sudan, and Kenya [36, 39, 47]. This might be due to the 


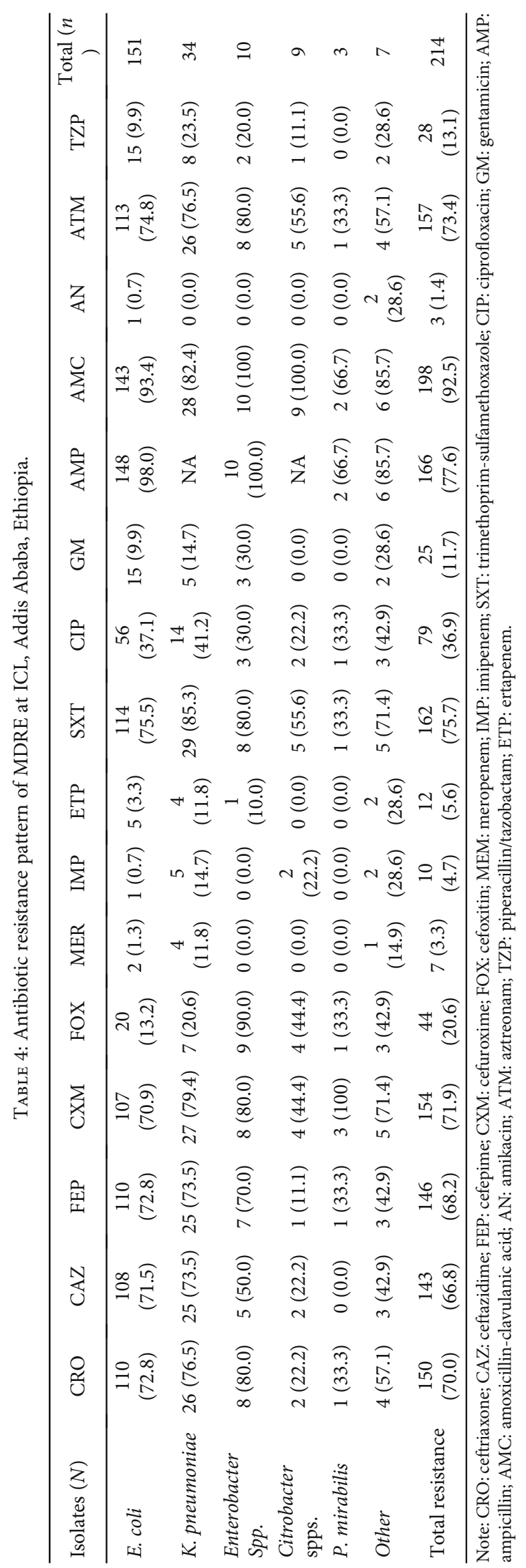




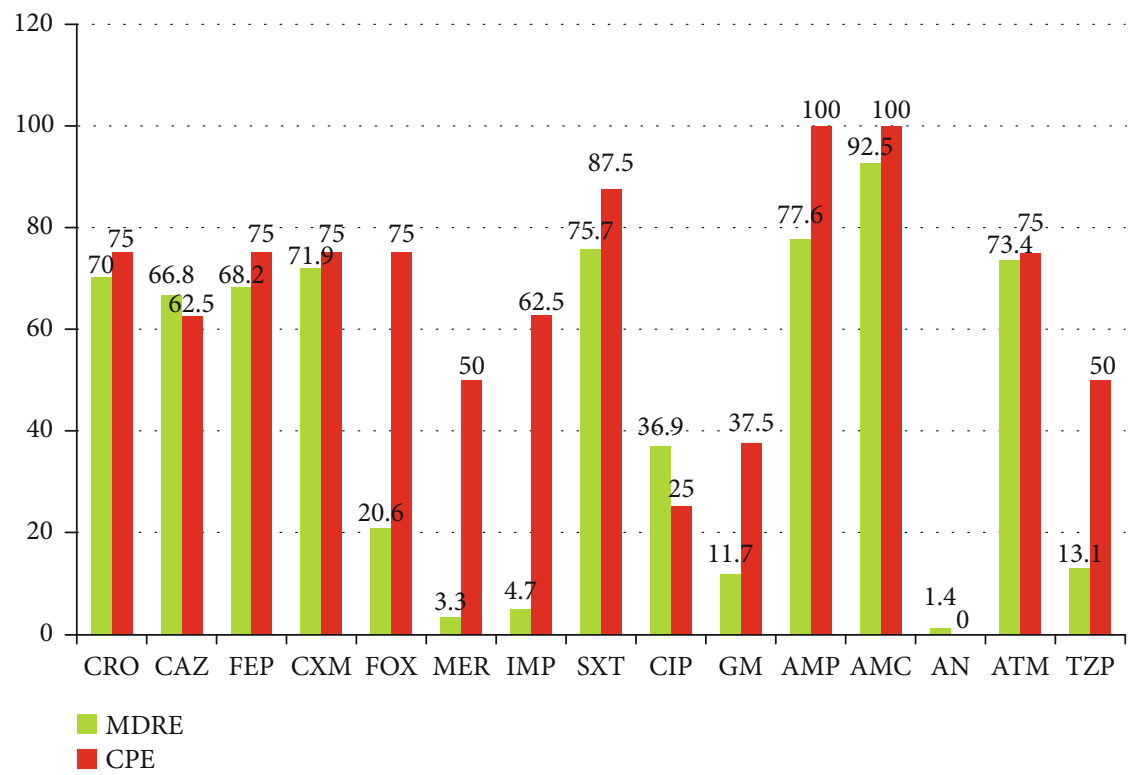

FIgURE 1: Antimicrobial resistance pattern of MDRE and CPE among clinical specimens at ICL, Addis Ababa, Ethiopia.

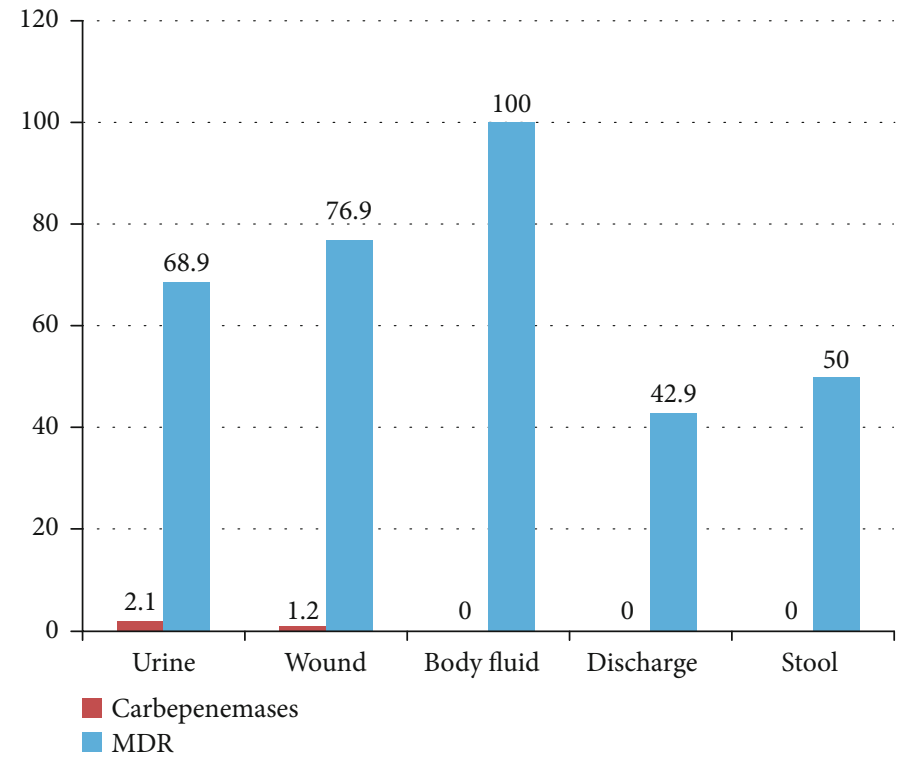

FIgURE 2: Distribution of carbapenemase-producing Enterobacteriaceae and MDR isolates among various clinical specimens at ICL, Addis Ababa, Ethiopia.

larger number of urine specimens included during the study period and also majority of study participants were females who are at high risk of infections especially urinary tract infection.

In the present study, the overall antibiotic resistance rates of carbapenemase-producing isolates were significantly higher for more than half of tested antibiotics including ampicillin (100\%), amoxicillin with clavulanic acid (100\%), sulfamethoxazole-trimethoprim (87.5\%), and ceftriaxone and cefepime (75.0\%). This finding was comparable with a study in Gondar: ampicillin (100\%), sulfamethoxazoletrimethoprim (100\%), amoxicillin with clavulanic acid
(100\%), ceftazidime (80\%), gentamycin (80\%), cefepime (60\%), and ceftriaxone (60\%) [22]; Jordan [35]; and Bangladesh [48] showing all carbapenemase-producing isolates indicating the highest resistance to amoxicillin with clavulanic acid (100\%), ampicillin (100\%), and cefepime (100\%). These findings indicated that carbapenemase-producing Enterobacteriaceae were the major cause of resistance to various antibiotic classes.

This study also showed that carbapenemase-producing Enterobacteriaceae were 100\% sensitive to amikacin and $75.0 \%$ to ciprofloxacin. This was fairly similar with a study conducted in Nigeria: ciprofloxacin (57.0\%) [49], Tanzania: 
ciprofloxacin (66.5\%) [50], and Nepal: amikacin (91.8\%) [20]. Antibiotic treatment options for carbapenem-resistant bacteria are limited and are highly costly. However, combination therapy with active drugs such as colistin, tigecycline, and fluoroquinolones can be alternative antibiotics [51].

\section{Conclusion and Recommendation}

In this study, a relatively higher prevalence of MDRE and significant prevalence of CPE were observed, and the highest resistance was recorded against ampicillin, amoxicillin with clavulanic acid, and sulfamethoxazole-trimethoprim. On the other hand, the better treatment option for CPE is amikacin and ciprofloxacin. The phenotypic confirmatory test indicated that more oxacillinase-48- (OXA-48-) producing Enterobacteriaceae was detected in this study. Detection of carbapenemase-producing Enterobacteriaceae is important for implementing appropriate antimicrobial therapy and in controlling the spread of the infection. Furthermore, continuous screening and investigations, including genotypic characterization of carbapenemase-producing Enterobacteriaceae, are required for the prevention and control of the spread of antimicrobial-resistant pathogens.

\section{Limitation of the Study}

(i) Molecular technique was not used for the characterization of carbapenemase-producingEnterobacteriaceae due to financial constraints.
Abbreviations
AST: $\quad$ Antibiotic susceptibility testing
ATCC: American Type Culture Collection
CLSI: Clinical and Laboratory Standards Institute
CRE: Carbapenem-resistant Enterobacteriaceae
CPE: $\quad$ Carbapenemase-producing Enterobacteriaceae
ESBL: $\quad$ Extended spectrum beta-lactamase
EUCAST: European Committee on Antimicrobial Suscep- tibility Testing
ICL: International Clinical Laboratories
IMP: Imipenemase
KPC: Klebsiella pneumoniae carbapenemase
MDR: Multidrug resistance
MIC: Minimum inhibitory concentration
MBL: $\quad$ Metallo beta-lactamase
mCIM: Modified carbapenem inactivation method
OXA-48: Oxacillinase-48 carbapenemase
VIM: $\quad$ Veronica integron metallo- $\beta$-lactamase types.

\section{Data Availability}

Data supporting the conclusion of this article are within the manuscript.

\section{Ethical Approval}

Ethical clearance was obtained from the research and ethical review committee of the Department of Medical Laboratory
Sciences, School of Allied Health Sciences, College of Health Sciences, Addis Ababa University, and an official permission letter was obtained from ICL.

\section{Conflicts of Interest}

The authors declare that they have no conflicts of interest in this research work.

\section{Authors' Contributions}

SG handled the study design, analysis, data collection, laboratory work, and interpretation of results, as well as writing of the manuscript; DS, DG, MA, SK, KD, and MH worked on advising and reviewing the manuscript. All authors read and approved the final manuscript.

\section{Acknowledgments}

The authors would like to acknowledge Addis Ababa University and International Clinical Laboratories for providing laboratory supplies.

\section{References}

[1] P. Nordmann, L. Dortet, and L. Poirel, "Carbapenem resistance in _Enterobacteriaceae_ : here is the storm!," Trends Mol Med, vol. 18, no. 5, pp. 263-272, 2012.

[2] J. Rodríguez-Baño, B. Gutiérrez-Gutiérrez, I. Machuca, and A. Pascual, "Treatment of infections caused by extended-spectrum-beta-lactamase-, AmpC-, and carbapenemaseproducing Enterobacteriaceae," Clinical microbiology reviews., vol. 31, no. 2, 2018.

[3] P. Nordmann, T. Naas, and L. Poirel, "Global spread of carbapenemase-producing Enterobacteriaceae," Emerging infectious diseases., vol. 17, no. 10, pp. 1791-1798, 2011.

[4] M. M. Tawfick, W. A. Alshareef, H. A. Bendary, H. Elmahalawy, and A. K. Abdulall, "The emergence of carbapenemase bla NDM genotype among carbapenem-resistant Enterobacteriaceae isolates from Egyptian cancer patients," European Journal of Clinical Microbiology \& Infectious Diseases., vol. 15, pp. 1-9, 2020.

[5] N. Kieffer, P. Nordmann, M. Aires-de-Sousa, and L. Poirel, "High prevalence of carbapenemase-producing Enterobacteriaceae among hospitalized children in Luanda, Angola," Antimicrobial agents and chemotherapy., vol. 60, no. 10, pp. 6189-6192, 2016.

[6] WHO, Report on antimicrobial resistance2017 [accessed 12 Oct 2017]. Available at http://www.who.int/mediacentre/ factsheets/fs194/en/].

[7] Centers for Disease Control and Prevention, "Vital signs: carbapenem-resistant Enterobacteriaceae," MMWR. Morbidity and Mortality Weekly Report, vol. 62, no. 9, pp. 165-170, 2013.

[8] Centers for Disease Control and Prevention (CDC), Antibiotic resistance threats in the United States, 2013, CDC, Atlanta, 2013, Available at: http://www.cdc.gov/drugresistance/threatreport-2013/pdf/ar-threats2013-508.pdf..

[9] A. Hossain, M. J. Ferraro, R. M. Pino et al., "Plasmid-mediated carbapenem hydrolyzing enzyme KPC-2 in an Enterobacter 
sp," Antimicrobial Agents and Chemotherapy, vol. 48, no. 11, pp. 4438-4440, 2004.

[10] A. Y. Peleg, C. Franklin, J. M. Bell, and D. W. Spelman, "Dissemination of the metallo-beta-lactamase gene blaIMP-4 among gram-negative pathogens in a clinical setting in Australia," Clinical Infectious Diseases, vol. 41, no. 11, pp. 1549-1556, 2005.

[11] A. Leavitt, S. Navon-Venezia, I. Chmelnitsky, M. J. Schwaber, and Y. Carmeli, "Emergence of KPC-2 and KPC-3 in carbapenem-resistant Klebsiella pneumoniae strains in an Israeli hospital," Antimicrobial Agents and Chemotherapy, vol. 51, no. 8, pp. 3026-3029, 2007.

[12] M. Akova, G. L. Daikos, L. Tzouvelekis, and Y. Carmeli, "Interventional strategies and current clinical experience with carbapenemase- producing Gram-negative bacteria," Clinical Microbiology and Infection, vol. 18, no. 5, pp. 439-448, 2012.

[13] BD Phoenix 100 Manual [20 Oct 2017]Available at: https:// www.bd.com//DS_IDS_Phoenix automated microbiologysys].

[14] CLSI, Performance Standards for Antimicrobial Susceptibility Testing; 26th Ed., CLSI Supplement M100S, Clinical and Laboratory Standards Institute, Wayne, PA, 2017.

[15] A. P. Magiorakos, A. Srinivasan, R. T. Carey et al., "Multidrugresistant, extensively drug-resistant and pandrug-resistant bacteria: an international expert proposal for interim standard definitions for acquired resistance," Clinical microbiology and infection., vol. 18, no. 3, pp. 268-281, 2012.

[16] Rosco KPC/MBL and OXA-48 insert kit [23 Oct 2017]Available at: http://www.eurobio.fr/images/Image/File/rosco/kit_esbl_ rosco.pdf].

[17] D. S. Teklu, A. A. Negeri, M. H. Legese, T. L. Bedada, H. K. Woldemariam, and K. D. Tullu, "Extended-spectrum betalactamase production and multi-drug resistance among Enterobacteriaceae isolated in Addis Ababa, Ethiopia," Antimicrobial Resistance \& Infection Control., vol. 8, no. 1, p. 39, 2019.

[18] F. M. G. Moges and G. Genetu, "Multiple drug resistance in urinary pathogens at Gondar College of Medical Sciences Hospital, Ethiopia," East Afric Med J, vol. 79, no. 8, pp. 415-420, 2002.

[19] W. Mulu, B. Abera, M. Yimer, T. Hailu, H. Ayele, and D. Abate, "Bacterial agents and antibiotic resistance profiles of infections from different sites that occurred among patients at Debre Markos Referral Hospital, Ethiopia: a cross-sectional study," BMC Research Notes, vol. 10, no. 1, p. 254, 2017.

[20] K. Nepal, N. D. Pant, B. Neupane et al., "Extended spectrum beta-lactamase and metallo beta-lactamase production among Escherichia coli and Klebsiella pneumoniae isolated from different clinical samples in a tertiary care hospital in Kathmandu, Nepal," Ann Clin Microbiol Antimicrob, vol. 16, no. 1, p. 62, 2017.

[21] D. Beyene, A. Bitew, S. Fantew, A. Mihret, and M. Evans, "Multidrug-resistant profile and prevalence of extended spectrum $\beta$-lactamase and carbapenemase production in fermentative Gram-negative bacilli recovered from patients and specimens referred to National Reference Laboratory, Addis Ababa, Ethiopia," PloS one., vol. 14, no. 9, article e0222911, 2019.

[22] S. Eshetie, C. Unakal, A. Gelaw, B. Ayelign, M. Endris, and F. Moges, "Multidrug resistant and carbapenemase producing Enterobacteriaceae among patients with urinary tract infection at referral hospital, Northwest Ethiopia," Antimicrob resist \& infect cont., vol. 4, no. 1, p. 12, 2015.

[23] B. Abera, M. Kibret, and W. Mulu, "Extended-spectrum betalactamases and antibiogram in Enterobacteriaceae from clinical and drinking water sources from Bahir Dar city, Ethiopia," PLoS One, vol. 11, no. 11, pp. 1-10, 2016.

[24] B. T. Van der Meeren, K. D. Chhaganlal, A. Pfeiffer et al., "Extremely high prevalence of multi-resistance among uropathogens from hospitalised children in Beira, Mozambique," South African Medical Journal., vol. 103, no. 6, pp. 382-386, 2013.

[25] T. A. Leski, C. R. Taitt, U. Bangura et al., "High prevalence of multidrug resistant Enterobacteriaceae isolated from outpatient urine samples but not the hospital environment in Bo, Sierra Leone," BMC Infectious Disease., vol. 16, no. 1, p. 167, 2016.

[26] H. Fazeli, S. Moghim, and D. Zare, "Antimicrobial resistance pattern and spectrum of multiple-drug-resistant enterobacteriaceae in Iranian hospitalized patients with cancer," Advanced Biomedical Research, vol. 7, 2018.

[27] K. K. Yadav, N. Adhikari, R. Khadka, A. D. Pant, and B. Shah, "Multidrug resistant Enterobacteriaceae and extended spectrum beta-lactamase producing Escherichia coli: a crosssectional study in National Kidney Center, Nepal," Antimicrob Res and Inf Cont, vol. 4, no. 1, p. 42, 2015.

[28] G. Godebo, G. Kibru, and H. Tassew, "Multidrug-resistant bacterial isolates in infected wounds at Jimma University Specialized Hospital, Ethiopia," Ann Cli Micro \& Ant., vol. 12, no. 1, p. 17, 2013.

[29] P. Baral, S. Neupane, B. P. Marasini, K. R. Ghimire, B. Lekhak, and B. Shrestha, "High prevalence of multidrug resistance in bacterial uropathogens from Kathmandu, Nepal," BMC Research notes., vol. 5, no. 1, p. 38, 2012.

[30] T. Khawcharoenporn, S. Vasoo, and K. Singh, "Urinary tract infections due to multidrug-resistant Enterobacteriaceae: prevalence and risk factors in a Chicago Emergency Department," Emergency medicine international., vol. 31, p. 2013, 2013.

[31] B. Lamichhane, C. Thakur, and S. K. Jain, "Antibiotic resistance patterns of gram negative isolates in a tertiary care hospital of Nepal," Asian Journal of Pharmaceutical and Clinical Research, vol. 7, no. 3, pp. 30-33, 2014.

[32] Z. Eslami Nejad, E. Ghafouri, Z. Farahmandi-Nia, B. Kalantari, and F. Saffari, "Isolation, identification, and profile of antibiotic resistance of bacteria in patients with cancer," Iran J Med Sci., vol. 35, pp. 109-115, 2015.

[33] M. A. Wartiti, F. Z. Bahmani, F. Elouennass, and A. Benouda, "Prevalence of carbapenemase- producing Enterobacteriaceae in a university hospital in Rabat, Morocco: a 19-months prospective study," Int Arabic J Antimicrob agents, vol. 2, no. 3, p. 4, 2012.

[34] C. C. Lai, U. I. Wu, J. T. Wang, and S. C. Chang, "Prevalence of carbapenemase-producing Enterobacteriaceae and its impact on clinical outcomes at a teaching hospital in Taiwan," Journal of the Formosan Medical Association., vol. 112, no. 8, pp. 492496, 2013.

[35] A. A. Aqel, P. Giakkoupi, H. Alzoubi, I. Masalh, M. J. Ellington, and A. Vatopoulos, "Detection of OXA-48-like and NDM carbapenemases producing _Klebsiella pneumoniae in Jordan: a pilot study," J. Infec and Public Health., vol. 10, no. 2, pp. 150-155, 2017.

[36] M. H. Legese, G. M. Weldearegay, and D. Asrat, "Extendedspectrum beta-lactamase- and carbapenemase-producing 
$<e m>$ Enterobacteriaceae $</ e m>$ among Ethiopian children," Infection and drug resistance., vol. Volume 10, pp. 27-34, 2017.

[37] D. Okoche, B. B. Asiimwe, F. A. Katabazi, L. Kato, and C. F. Najjuka, "Prevalence and characterization of carbapenemresistant Enterobacteriaceae isolated from Mulago National Referral Hospital, Uganda," PLOS One, vol. 10, no. 8, pp. 110, 2015.

[38] R. Tewari, S. D. Mitra, F. Ganaie et al., "Prevalence of extended spectrum $\beta$-lactamase, AmpC $\beta$-lactamase and metallo $\beta$-lactamase mediated resistance in Escherichia coli from diagnostic and tertiary healthcare centers in South Bangalore, India," Int $J$ Res Med Sci., vol. 6, no. 4, pp. 1308-1313, 2018.

[39] R. A. Dahab, A. M. Ibrahim, and H. N. Altayb, "Phenotypic and genotypic detection of carbapenemase enzymes producing gram negative bacilli isolated from patients in Khartoum State," F1000Research, vol. 7, p. 6, 2017.

[40] R. I. Manenzhe, H. J. Zar, and M. P. Nicol, "The spread of carbapenemase-producing bacteria in Africa: a systematic review," J Antimicrob Chemo., vol. 70, no. 1, pp. 23-40, 2015.

[41] N. A. Kamel, W. N. El-Tayeb, M. R. El-Ansary, M. T. Mansour, and K. M. Aboshanab, "Phenotypic screening and molecular characterization of carbapenemase-producing Gramnegative bacilli recovered from febrile neutropenic pediatric cancer patients in Egypt," PLoS One, vol. 13, no. 8, article e0202119, 2018.

[42] M. M. Gómara-Lomero, A. I. López-Calleja, B. M. Vela Iglesia, I. F. Cerón, A. R. López, and M. J. Pinilla, "Deteccion de carbapenemasas y otros mecanismos de resistencia enzimaticos a $\beta$ lactamicos en enterobacterias con sensibilidad disminuida a carbapenems en un hospital de tercer nivel," Enferm Infec Microbiol Clin., vol. 36, no. 5, pp. 296-301, 2018.

[43] B. Bedeni, V. PleIko, S. Sardeli, S. Uzunovi, and K. G. Torkar, "Carbapenemases in Gram negative bacteria," Lab Detect \& Clinic Signif, vol. 3, 2014.

[44] O. O. Oduyebo, O. M. Falayi, P. Oshun, and A. O. Ettu, "Phenotypic determination of carbapenemase producing Enterobacteriaceae isolates from clinical specimens at a tertiary hospital in Lagos, Nigeria," Niger Postgrad Med J, vol. 22, no. 4, pp. 223-227, 2015.

[45] W. Laolerd, Y. Akeda, L. Preeyanon, P. Ratthawongjirakul, and P. Santanirand, "Carbapenemase-Producing CarbapenemResistantEnterobacteriaceaefrom Bangkok, Thailand, and their detection by the Carba NP and Modified carbapenem inactivation Method Tests," Microbial Drug resistance, vol. 24, no. 7, pp. 1006-1011, 2018.

[46] A. Amjad, I. A. Mirza, S. A. Abbasi, U. Farwa, N. Malik, and F. Zia, "Modified Hodge test: a simple and effective test for detection of carbapenemase production," Iran. J. Microbiol., vol. 3, no. 4, pp. 189-193, 2011.

[47] H. I. Magale, I. A. Kassim, S. A. Odera, M. J. Omolo, W. G. Jaoko, and P. E. Jolly, "Antibiotic susceptibility of organisms causing urinary tract infection in patients presenting at Kenyatta National Hospital, Nairobi," East Afr Med J, vol. 2, p. 5, 2016.

[48] N. Hayder, Z. Hasan, S. Afrin, and R. Noor, "Determination of the frequency of carbapenemase producing Klebsiella pneumoniae isolates in Dhaka city, Bangladesh," Stamford J Microbiol, vol. 2, no. 1, pp. 2074-5346, 2012.

[49] Y. Ibrahim, Y. Sani, Q. Saleh, A. Saleh, and G. Hakeem, "Phenotypic detection of extended spectrum beta-lactamase and carbapenemase co-producing clinical isolates from two ter- tiary hospitals in Kano, North West Nigeria," Ethiopian Journal of Health Sciences, vol. 27, no. 1, p. 3010, 2017.

[50] M. F. Mushi, S. E. Mshana, C. Imirzalioglu, and F. Bwanga, "Carbapenemase genes among multidrug resistant gram negative clinical isolates from a tertiary hospital in Mwanza, Tanzania," BioMed Research International, vol. 2014, 2014.

[51] S. S. Kanj and Z. A. Kanafani, "Current concepts in antimicrobial therapy against resistant Gram-negative organisms: extended-spectrum $\beta$-lactamase-producing Enterobacteriaceae, carbapenem-resistant Enterobacteriaceae, and multidrugresistant_Pseudomonas aeruginosa_," Mayo Clinic Proceedings, vol. 86, no. 3, pp. 250-259, 2011. 